Geophysical and Astrophysical Fluid Dynamics

Vol. 00, No. 00, 00 Month 2017, 1-11

\title{
Dynamos in an annulus with fields linear in the axial coordinate
}

\author{
R. H. VAZ*, F. A. T. BOSHIER and A. J. MESTEL \\ Mathematics Department, Imperial College London, London, UK \\ (v4.4 released October 2012)
}

\begin{abstract}
Dynamo action is considered in a conducting cylindrical annulus surrounded by an insulator. The driving velocity field is assumed to be linear in the axial coordinate and to satisfy the incompressible Navier-Stokes equations. Such flows have recently been shown to exist with no forcing other than the similarity structure. Magnetic field instabilities with the same spatial structure are sought. The kinematic eigenvalue problem is found to have two growing modes for moderate values of the magnetic Reynolds number, $R_{m}$. As $R_{m} \rightarrow \infty$ it is shown that the modes are governed by layers on the outer wall. The growing field saturates in a solution to the nonlinear dynamo problem. Three distinct steady solution families are found and the complicated bifurcation structure is investigated.
\end{abstract}

Keywords: Dynamo; Annulus; Similarity solutions

\section{Introduction}

The generation of a magnetic field by the motion of electrically conducting fluids is important in many geophysical and astrophysical problems. An extensive review of the subject can be found in Roberts and Soward (1992). Simple analytical models, especially those which extend into the nonlinear regime, are in short supply. One of the simplest dynamos is the discontinuous Ponomarenko flow, consisting of the constant rotation and translation of a fluid cylinder surrounded by stationary insulator. This dynamo is fast, in that the growth rate has a non-zero limit as $R_{m} \rightarrow \infty$. Generalisations of this flow to continuous velocity fields varying only with radius were considered by Ruzmaikin et al. (1988) and Gilbert (1988). These dynamos were shown to be slow, decaying as $R_{m}^{-1 / 3}$ as $R_{m} \rightarrow \infty$. Flows of this type satisfy the Euler equations. Dynamos for the Taylor-Couette flow satisfying the Navier-Stokes solutions were found by Bassom and Gilbert (1997) in a cylinder and by Wynne (2017) in an annulus.

More general velocity fields could be expected to vary in the axial direction. Recently the authors have shown that the incompressible Navier-Stokes equations have solutions in a stationary cylindrical annulus of the form

$$
\boldsymbol{u}=\left(-\frac{\psi(r)}{r}, y w(r), y \frac{\psi^{\prime}(r)}{r}\right) \quad \text { in } a<r<b
$$

in terms of cylindrical coordinates $(r, \theta, y)$, (Vaz et al. 2018). These similarity flows have no explicit forcing and exist due to the unbounded nature of the domain in the $y$-direction. For a fixed value of the annulus aspect ratio, $b / a$, two such solutions were found, although two additional solutions with $w=0$ exist for sufficiently high $b / a$. In this paper we investigate whether such velocity fields can drive dynamo action. We shall concentrate almost exclusively on one particular solution for the annulus $1<r<3$.

*Corresponding author. Email: r.vaz14@imperial.ac.uk 
We shall not consider all possible dynamo modes. Rather, we investigate fields with the same spatial similarity structure as the velocity field. This reduces the problem to a set of coupled ODEs. It has the further advantage that we can readily consider nonlinear interactions of the Lorentz force with the (laminar) flow. Since both velocity and magnetic fields are axisymmetric it might be expected that dynamo action would be ruled out by Cowling's theorem Cowling (1933). However this is not the case because of the unbounded domain and its associated infinite energy. Our similarity structure permits advection from $y= \pm \infty$. Indeed, we even find unforced viscous flows as in Vaz et al. (2018).

The outline of this paper is as follows. In section 2 we derive the governing equations for the kinematic dynamo in the annulus. In section 3 we investigate the range of $R_{m}$ for which unstable modes exist. Two distinct modes are found. For large $R_{m}$, both modes are shown to be governed by the outer wall behaviour. Curiously, as $a / b \rightarrow 0$, and the flow develops a stress singularity on the axis, a fast dynamo is approached, in a manner reminiscent of the Ponomarenko dynamo.

In section 4 steady solutions of the fully nonlinear system are sought. Several solution branches are found. Surprisingly, many of the solution branches cease to exist as $R_{m}$ increases, and nonlinear subcritical solutions exist as $R_{m} \rightarrow 0$. In this limit $R_{m}$ should really be interpreted as the magnetic Prandtl number and the flow is driven by the magnetic rather than hydrodynamic boundary conditions. We conclude in section 5 .

\section{Governing equations}

We use cylindrical polar coordinates $(r, \phi, y)$ and work in the annular domain $a<r<b$ as depicted in figure 1. In kinematic dynamo theory the velocity field is regarded as given and self-excited solutions of the magnetic induction equations are sought (Roberts and Soward 1992). In non-dimensional form, using scales $L, U_{0}$ and $L / U_{0}$ for length, velocity and time respectively, these are given by

$$
\frac{\partial \boldsymbol{B}}{\partial t}=\boldsymbol{\nabla} \times(\boldsymbol{u} \times \boldsymbol{B})+R_{m}^{-1} \nabla^{2} \boldsymbol{B}, \quad \boldsymbol{\nabla} \cdot \boldsymbol{B}=0 .
$$

Here $\boldsymbol{B}$ is the magnetic field, $t$ is time, $\boldsymbol{u}$ the velocity field and $R_{m}=U_{0} L / \eta$ is the magnetic Reynolds number.

We shall use for the velocity field, $\boldsymbol{u}$, an unforced solution of the steady Navier-Stokes in an infinite annulus as reported in Vaz et al. (2018). As there is no explicit forcing term, it is natural to use the velocity scale $U_{0}=\nu / L$, where $\nu$ is the kinematic viscosity, so that $\boldsymbol{u}$ satisfies

$$
\boldsymbol{u} \cdot \boldsymbol{\nabla} \boldsymbol{u}=-\boldsymbol{\nabla} p+\nabla^{2} \boldsymbol{u}, \quad \boldsymbol{\nabla} \cdot \boldsymbol{u}=0,
$$

with the solid body boundary conditions $\boldsymbol{u}=0$ on $r=a, b$. For the length scale, $L$, we shall use the annulus halfwidth, so that the fluid domain is $a<r<b \equiv a+2$, where $a$ is now nondimensional; for convenience we shall continue to refer to $b$ below. A consequence of scaling the velocity field with $\nu / L$ is that the magnetic Reynolds number can also be regarded as a magnetic Prandtl number, $R_{m}=\nu / \eta$.

In Vaz et al. (2018), it was shown that (3a,b) possess a few non-trivial solutions of the form

$$
\boldsymbol{u}=\left(-\frac{\psi(r)}{r}, y w(r), y \frac{\psi^{\prime}(r)}{r}\right),
$$

where $y \psi(r)$ is a Stokes streamfunction and $y w(r)$ the velocity in the $\phi$-direction. Assuming there is no azimuthal pressure gradient the $\phi$-component of (3a) becomes

$$
-\psi w^{\prime}+\psi^{\prime} w-\frac{\psi w}{r}=w^{\prime \prime} r+w^{\prime}-\frac{w}{r},
$$


and taking the curl to eliminate pressure gives

$$
-\psi^{\prime \prime \prime} \psi+\psi^{\prime \prime} \psi^{\prime}+3 \frac{\psi^{\prime \prime} \psi}{r}-\frac{\left(\psi^{\prime}\right)^{2}}{r}-3 \frac{\psi^{\prime} \psi}{r^{2}}+2 w^{2} r=\psi^{\prime \prime \prime \prime} r-2 \psi^{\prime \prime \prime}+3 \frac{\psi^{\prime \prime}}{r}-3 \frac{\psi^{\prime}}{r^{2}} .
$$

The no-slip condition requires

$$
w=\psi=\psi^{\prime}=0 \quad \text { at } \quad r=a, b .
$$

In this paper, unless stated otherwise, we shall fix $a=1$ and $b=3$, values chosen so that

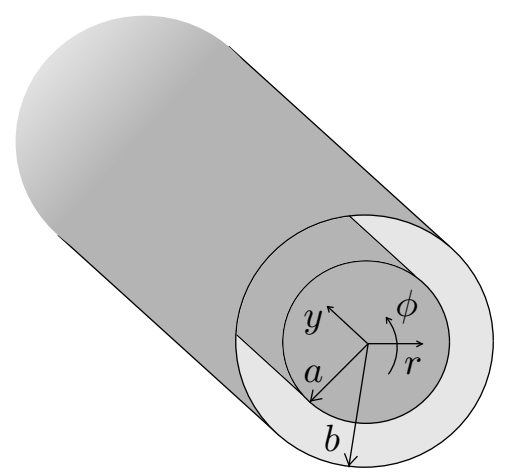

Figure 1. Depiction of geometry and coordinate system $(r, \phi, y)$, for the infinite annulus, $a<r<b$.

the curvature is $\mathrm{O}(1)$. In Vaz et al. (2018) we found two non-trivial solutions of (5) and (6) in this geometry. In the interest of conciseness we consider only one such solution here, which is shown in figure 2. We shall seek solutions for the magnetic field with a similar spatial
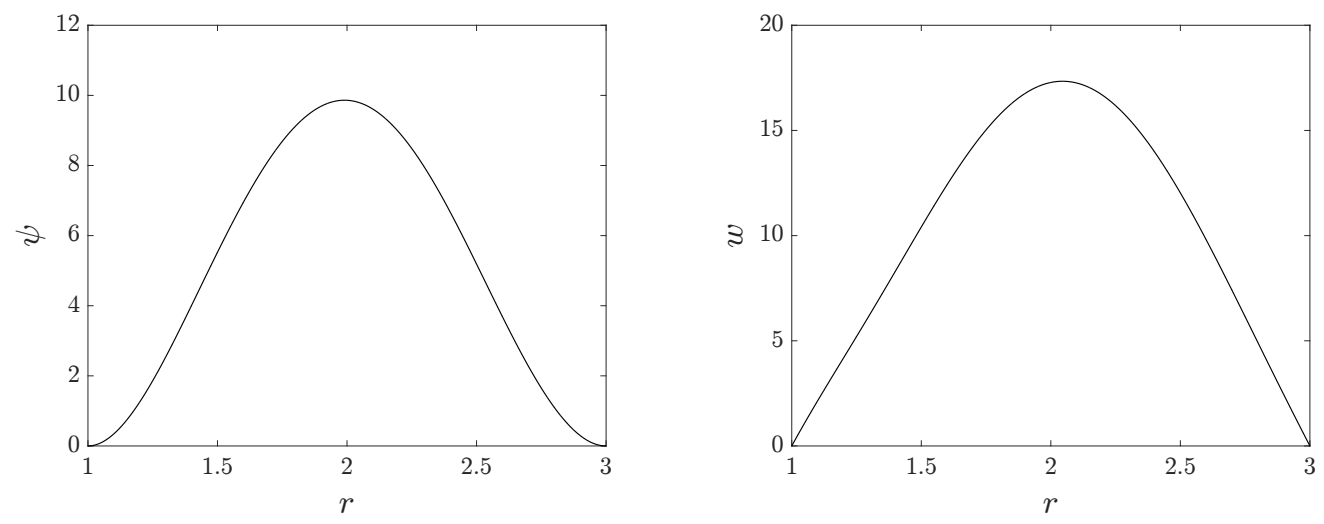

Figure 2. Unforced velocity field in an infinite annulus with $a=1$ and $b=3$.

structure, defining a flux function, $y \chi(r)$, and an azimuthal field $y C(r)$ analagous to $\psi$ and $w$,

$$
\boldsymbol{B}=\left(-\frac{\chi(r)}{r}, y C(r), y \frac{\chi^{\prime}(r)}{r}\right) .
$$

This magnetic field has a nondimensional current density $\boldsymbol{j}=\boldsymbol{\nabla} \times \boldsymbol{B}$ given by

$$
\boldsymbol{j}=\left(-C(r),-y\left(\frac{\chi^{\prime}(r)}{r}\right)^{\prime}, y \frac{(r C(r))^{\prime}}{r}\right) .
$$


Since for the kinematic problem we regard $w$ and $\psi$ as given, the system of equations is linear. We can look for exponential time behaviour $\boldsymbol{B} \propto \mathrm{e}^{\lambda t}$, where for a dynamo to exist, $\operatorname{Re}\{\lambda\}>0$. Our system of equations reduces to the eigenvalue problem,

$$
\begin{aligned}
\lambda \chi & =-\frac{\psi^{\prime} \chi}{r}+\frac{\psi \chi^{\prime}}{r}+R_{m}^{-1}\left(\chi^{\prime \prime}-\frac{\chi^{\prime}}{r}\right), \\
\lambda C & =\frac{w \chi^{\prime}}{r}-\frac{\psi^{\prime} C}{r}+\frac{\psi C^{\prime}}{r}-\frac{w^{\prime} \chi}{r}+\frac{w \chi}{r^{2}}-\frac{\psi C}{r^{2}}+R_{m}^{-1}\left(C^{\prime \prime}+\frac{C^{\prime}}{r}-\frac{C}{r^{2}}\right),
\end{aligned}
$$

where the primes denote a derivative with respect to $r$. The absence of $y$ illustrates the consistency of the ansatz $(4,8)$. We note that (10a), which determines $\chi$, is independent of $C$ and $w$. This is a familiar phenomenon in dynamo theory - while differential rotation can generate toroidal field from poloidal field, it is frequently difficult to drive poloidal field. Thus in the Ponomarenko dynamo, for example, a growing magnetic field necessarily varies with the toroidal coordinate. Here, the linear variation in $y$ permits an axisymmetric field.

In our analysis we consider that the region outside the annulus is insulating. In a perfect insulator there is no current, hence $\boldsymbol{j}=\mathbf{0}$ in $r<a$ and $r>b$. So for $r<a$, using (9), we require $C=0$ and $\chi=A_{1}+B_{1} r^{2}$, and for regularity at $r=0$ we must impose $A_{1}=0$. Outside the outer wall, $r>b$, the solution has the same structure, $C=0$ and $\chi=A_{2}+B_{2} r^{2}$. For a zero field as $r \rightarrow \infty$ we impose $B_{2}=0$. Thus we permit a stagnation point field in $r<a$ and a decaying radial field in $r>b$ if required by the similarity solution in the annulus. In order to match with these external magnetic fields our boundary conditions are

$$
\begin{aligned}
C=0 \quad \text { at } & r=a, b, \\
\left(\frac{\chi}{r^{2}}\right)^{\prime}=0 \quad \text { at } \quad & r=a, \\
\chi^{\prime}=0 \quad \text { at } & r=b .
\end{aligned}
$$

\section{Kinematic dynamos}

We discretise (10), using second order centred finite differences and use $N \geqslant 4000$ mesh points. We then solve the eigenvalue problem,

$$
\mathrm{M} \Phi_{i}=\lambda_{i} \Phi_{i} .
$$

where $\mathrm{M}$ is the $2 N \times 2 N$ coefficient matrix, $\lambda_{i}$ the eigenvalues, and $\Phi_{i}$ the corresponding eigenvector that yields the mode-shape of $\chi$ and $C$. Positive $\operatorname{Re}\{\lambda\}$ values translate to selfexciting modes, i.e., a dynamo.

We prescribe the velocity field shown in figure 2, and impose perfect insulator boundary conditions (11). We find two linearly unstable modes, both with real $\lambda$. The growth rates as functions of $R_{m}$ are presented in figure 3. The modes are decoupled, as can be predicted from (10). One mode has $\chi \equiv 0$ while $C$ is determined by (10b). This mode, which we call the $C$-mode, is therefore independent of $w$. It becomes unstable at $R_{m} \simeq 2.19$ reaching a maximum $\lambda_{\max } \simeq 1.39$ at $R_{m} \simeq 15.17$. The $C$-mode is presented in figure 4 for these values. The growth rate $\lambda$ of the other mode is determined solely by $\chi(10 \mathrm{a})$. We call this the $\chi$-mode, as although $C$ is non-zero, it is passive, driven by (10b). The $\chi$-mode first goes unstable at $R_{m} \simeq 0.17$ reaching a maximum $\lambda_{\max } \simeq 4.13$ at $R_{m} \simeq 1.69$. It is drawn in figure 4 for these values.

Both modes are found numerically to decay with $\lambda \sim R_{m}^{-1 / 3}$ as $R_{m} \rightarrow \infty$. We analyse this asymptotic behaviour, anticipating boundary layers on the walls. For illustration, we consider 
the $\chi$-mode on the outer wall, but the analysis is similar for the $C$-mode and for the inner wall. Since $\mathbf{u}$ satisfies the no-slip condition, near the wall we have $\psi \sim \alpha x^{2}$, where near the outer wall $x=b-r$. Performing the change of variable,

$$
z=(b-r) R_{m}^{1 / 3}
$$

and substituting this into (10a), and assuming that $R_{m}^{1 / 3} \gg 1$, we obtain at leading order

$$
\mu \chi=-\frac{\alpha}{b} z^{2} \chi^{\prime}+\frac{2 \alpha}{b} z \chi+\chi^{\prime \prime}
$$

where $\mu=\lambda R_{m}^{1 / 3}$, and the primes denote a derivative with respect to $z$. As $z \rightarrow \infty$, this linear equation has a solution for which at leading order $\chi^{\prime} \sim \exp \left(\alpha z^{3} / 3 b\right)$ and another for which $\chi \sim z^{2}$. If $\alpha<0$, there is no difficulty matching with the core for any value of $\mu$. However, if $\alpha>0$, a solution can only be found if the coefficient of the exponentially growing solution vanishes. This happens only for special values of the eigenvalue $\mu$. For our flow $\alpha>0$ at the outer wall, but the equivalent problem at the inner wall has no difficulty. The eigenvalue is thus determined by the behaviour at the outer wall. We therefore impose on (14)

$$
\begin{aligned}
\chi^{\prime} & =0 \quad \text { at } \quad z=0, \\
\left(\frac{\chi}{z^{2}}\right)^{\prime} & =0 \quad \text { as } \quad z \rightarrow \infty
\end{aligned}
$$

and solve the eigenvalue problem numerically for the appropriate value of $\alpha / b$. The $C$-mode satisfies the same ODE near the outer wall, but has a different boundary condition, $C=0$ at $z=0$, so that the eigenvalue differs. The full numerical and asymptotic solutions for both cases agree well as can be seen in figure 3. This yields two interesting results. As $R_{m} \rightarrow \infty$, even though the field is largest away from the walls, its growth rate is determined by a boundary layer on the wall from which the radial flow is directed away from the wall. Secondly, this analysis applies for any velocity field. All viscous flows satisfy no-slip at the wall, hence $\psi \sim \alpha x^{2}$ is perfectly general.

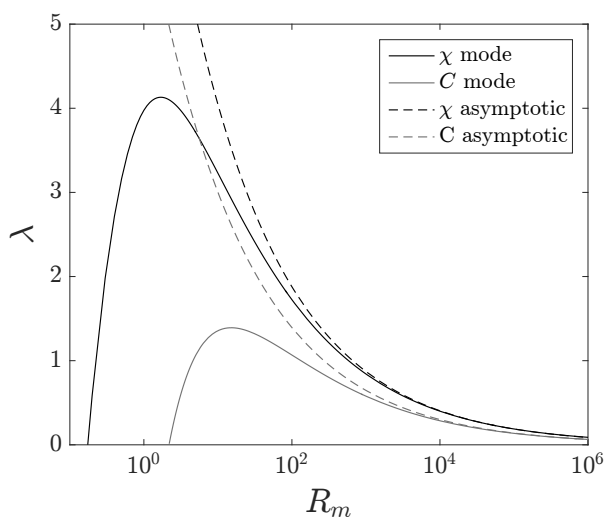

Figure 3. Growth rate of the two unstable modes as a function of $R_{m}$. The $C$-mode goes unstable at $R_{m} \simeq 2.19$ and reaches a maximum $\lambda_{\max } \simeq 1.39$ at $R_{m} \simeq 15.17$. The $\chi$-mode becomes unstable at a $R_{m} \simeq 0.17$ reaching a maximum $\lambda_{\max } \simeq 4.13$ at $R_{m} \simeq 1.69$. Both modes exhibit a decay rate $\lambda \sim R_{m}^{-1 / 3}$ as $R_{m} \rightarrow \infty$, with a coefficient predicted by a local eigensolution of the outer layer.

Digressing slightly, in Vaz et al. (2018) additional unforced flows were found that exist only when $a \ll b$. These flows are poloidal as $w \equiv 0$. Defining the annulus curvature, $\delta$, as the ratio of the gap size to the mid-radius,

$$
\delta=\frac{2}{1+a},
$$



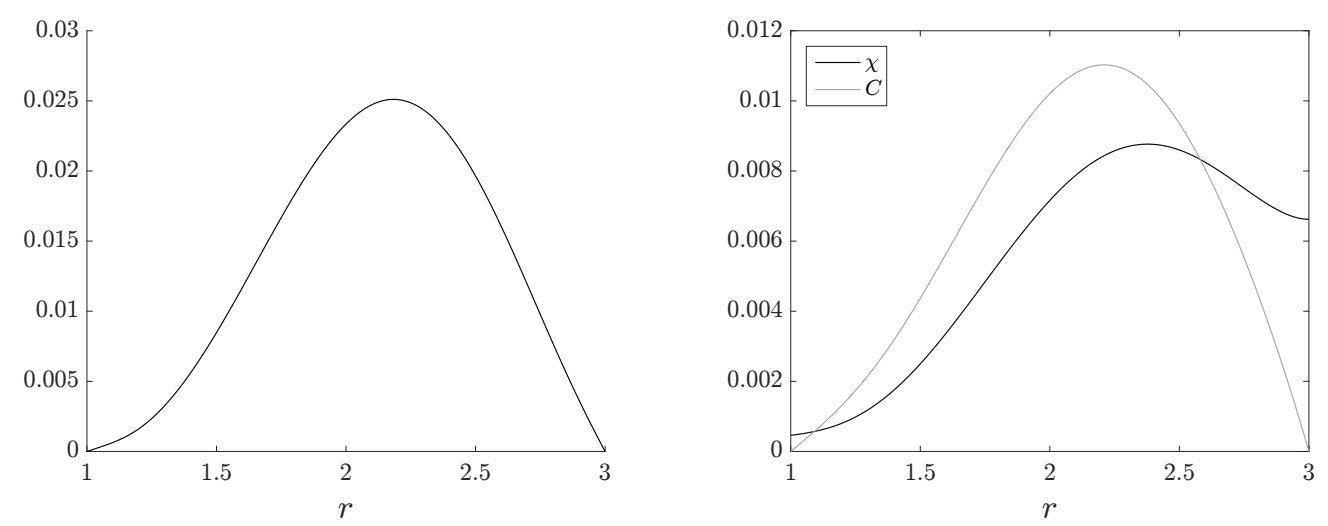

Figure 4. $C$-mode on the left with $\chi \equiv 0$, and $\chi$ mode with $C$ passive on the right. These modes have been obtained for $\lambda_{\max }$ in each case.

so that $0<\delta<2$, we choose one such flow for $\delta=1.998$, depicted in figure 5 . It is found to drive two dynamos, one with $C \equiv 0$ and the other with $\chi \equiv 0$. In figure 5 we plot both growth rates as functions of $R_{m}$. The decay as $R_{m} \rightarrow \infty$ is markedly weaker for this small $a$-value than, say, in figure 3. Indeed, for fixed $R_{m} \gg 1$, as $a \rightarrow 0$ and $\delta \rightarrow 2$, the growth rates tend to a constant, indicating that the dynamos are fast. This limit is not the same as removing the inner cylinder, as we are imposing no-slip on $r=a \rightarrow 0$. The limiting flow has a stress singularity on the axis. This behaviour is reminiscent of the fast dynamo for the discontinuous Ponomarenko flow, which becomes slow when the singularity is smoothed out.
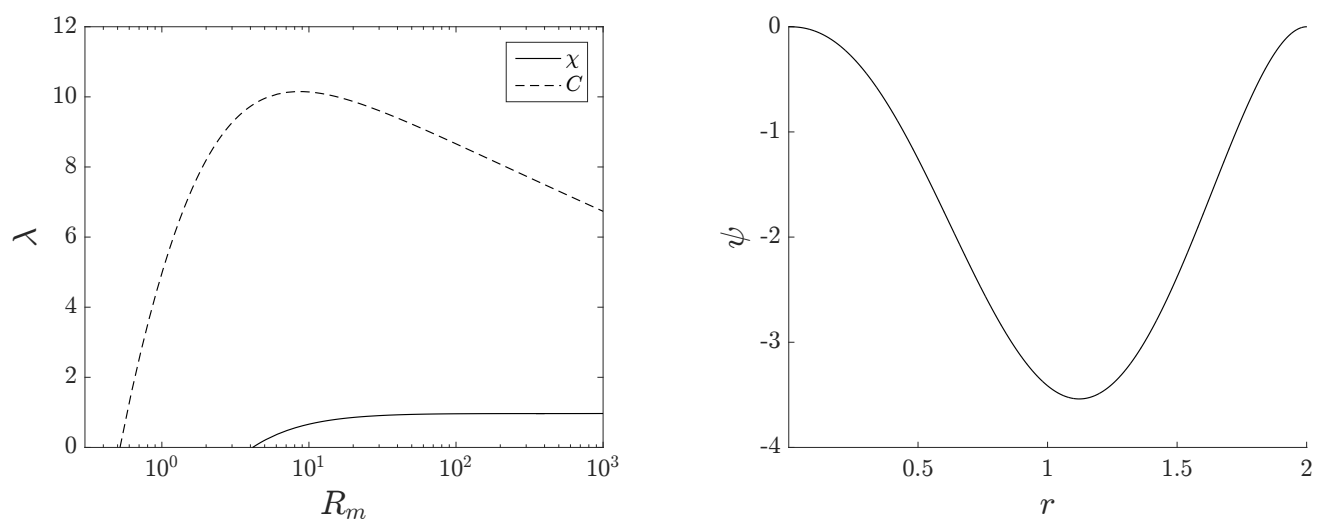

Figure 5. Growth rate of slowly decaying dynamo modes as a function of $R_{m}$ for a curvature $\delta=1.998$ and a flow with $w \equiv 0$ (right). As $\delta \rightarrow 2$, the flow develops a singularity and the dynamo becomes fast.

\section{Nonlinear system}

Having found growing solutions to the kinematic system, it is natural to consider the nonlinear dynamo, when the magnetic field reacts back on the driving flow. Indeed, this is a major reason for seeking solutions with a similarity structure. We investigate the existence of equilibrium states for the 4-dimensional system when $w$ and $\psi$ couple to $\chi$ and $C$. The coupling term is the Lorentz force $\boldsymbol{j} \times \boldsymbol{B}$ and the steady Navier-Stokes equations become

$$
\boldsymbol{u} \cdot \boldsymbol{\nabla} \boldsymbol{u}=-\nabla p+\boldsymbol{j} \times \boldsymbol{B}+\nabla^{2} \boldsymbol{u}, \quad \boldsymbol{\nabla} \cdot \boldsymbol{u}=0 .
$$


Assuming $\boldsymbol{u}$ and $\boldsymbol{B}$ to be given by (4) and (8), respectively, the full system of equations is

$$
\begin{aligned}
\psi^{\prime} \chi-\psi \chi^{\prime} & =R_{m}^{-1}\left(\chi^{\prime \prime} r-\chi^{\prime}\right), \\
-w \chi^{\prime}+\psi^{\prime} C-\psi C^{\prime}+w^{\prime} \chi-\frac{w \chi}{r}+\frac{\psi C}{r} & =R_{m}^{-1}\left(C^{\prime \prime} r+C^{\prime}-\frac{C}{r}\right), \\
-w^{\prime} \psi+w \psi^{\prime}-\frac{w \psi}{r}-\chi^{\prime} C+C^{\prime} \chi+\frac{C \chi}{r} & =w^{\prime \prime} r+w^{\prime}-\frac{w}{r}, \\
-\psi \psi^{\prime \prime \prime}+\psi^{\prime} \psi^{\prime \prime}-\frac{\left(\psi^{\prime}\right)^{2}}{r}+3 \frac{\psi^{\prime \prime} \psi}{r}-3 \frac{\psi^{\prime} \psi}{r^{2}}+2 w^{2} r & \\
+\chi \chi^{\prime \prime \prime}-\chi^{\prime} \chi^{\prime \prime}+\frac{\left(\chi^{\prime}\right)^{2}}{r}-3 \frac{\chi^{\prime \prime} \chi}{r}+3 \frac{\chi^{\prime} \chi}{r^{2}}-2 C^{2} r & =\psi^{\prime \prime \prime \prime} r-2 \psi^{\prime \prime \prime}+3 \frac{\psi^{\prime \prime}}{r}-3 \frac{\psi^{\prime}}{r^{2}},
\end{aligned}
$$

with the insulating and no-slip boundary conditions

$$
\begin{aligned}
C=0, \quad \psi=0, \quad w & =0 \quad \text { at } \quad r=a, b, \\
\left(\frac{\chi}{r^{2}}\right)^{\prime} & =0 \quad \text { at } \quad r=a, \\
\chi^{\prime} & =0 \quad \text { at } \quad r=b .
\end{aligned}
$$

This system is unforced, in that there is a solution with all fields zero. To seek a non-trivial solution numerically, we add a constant fictitious forcing $F$ to the right-hand-sides of some or all of equations (18) and solve the forced system for a fixed value of $R_{m}$. We tested forcing different combinations of equations aiming to find a solution branch with a turning point that could be continued back to $F=0$ unveiling saturated states of the system. For example, forcing solely (18b) and (18d) revealed a decoupled saturated state with $C$ and $\psi$ non-trivial but $\chi=w \equiv 0$, which we discuss below. Once a non-zero solution is located for $F=0$, we perform path-continuation to follow the solution branches as $R_{m}$ varies. Path-continuation methods are well reported in the literature, for example Chan (1984), Allgower and Georg (1993). Other types of forcing, for example, if $F$ were to be a function of $r$, could perhaps reveal additional unforced solutions to the ones we report here. Nonetheless, this method allows us to find the two solutions predicted by linear theory as well as some additional ones.

The equations possess a few obvious symmetries. They are unchanged if $(C, w) \rightarrow(-C,-w)$ (skew-symmetry) or if $(\chi, C) \rightarrow(-\chi,-C)$ (field reversal.) Solutions might be possible with all 4 fields non-zero, as well as solutions with one or more variables vanish. By inspection, in this latter case we might expect solutions where the non-vanishing fields are $\psi$ only, $(\psi, w)$, $(\psi, w, C),(\psi, C)$ or $(\psi, \chi)$. We do not find any poloidal solutions with only $\psi$ and $\chi$ at this curvature $(\delta=1)$. The hydrodynamic solutions, i.e. no magnetic field, are discussed in (Vaz et al. 2018), so we will not expand on them here. We start by analysing the behaviour of a decoupled solution family, where $\psi$ and $C$ are non-zero but $\chi=w \equiv 0$. On the right of figure 6 the solution profiles are drawn for $R_{m}=0.5$. We follow this solution as $R_{m}$ varies, and plot the $L^{\infty}$-norm of the variables in figure 6 . We find that $\psi \sim R_{m}^{-1}$ for all $R_{m}$, whereas $C$ has two distinct regimes, $C \sim R_{m}^{-1}$ as $R_{m} \rightarrow 0$ and $C \sim R_{m}^{-1 / 2}$ as $R_{m} \rightarrow \infty$. While it is at first surprising to get a dynamo-like solution for $R_{m} \ll 1$, we recall that $R_{m}$ is also the magnetic Prandtl number since the velocity field for this unforced flow is effectively scaled with the Reynolds number. It is perhaps misleading to regard these as dynamo modes. What is happening is that the similarity structure is permitting magnetic field to be drawn in from $y \pm \infty$, which then drives a flow balancing the rotational Lorentz force. Also, although it appears that the fields are large in this limit, that is an artefact of having used the velocity scale $\nu / L$ rather than $\eta / L$ which is more appropriate in this limit.

Another solution type we find has $\psi, w$ and $C$ non-zero and $\chi \equiv 0$. This solution branch starts at $R_{m}=2.19$, as predicted by the linear theory. As $R_{m}$ increases, it reaches a turning 

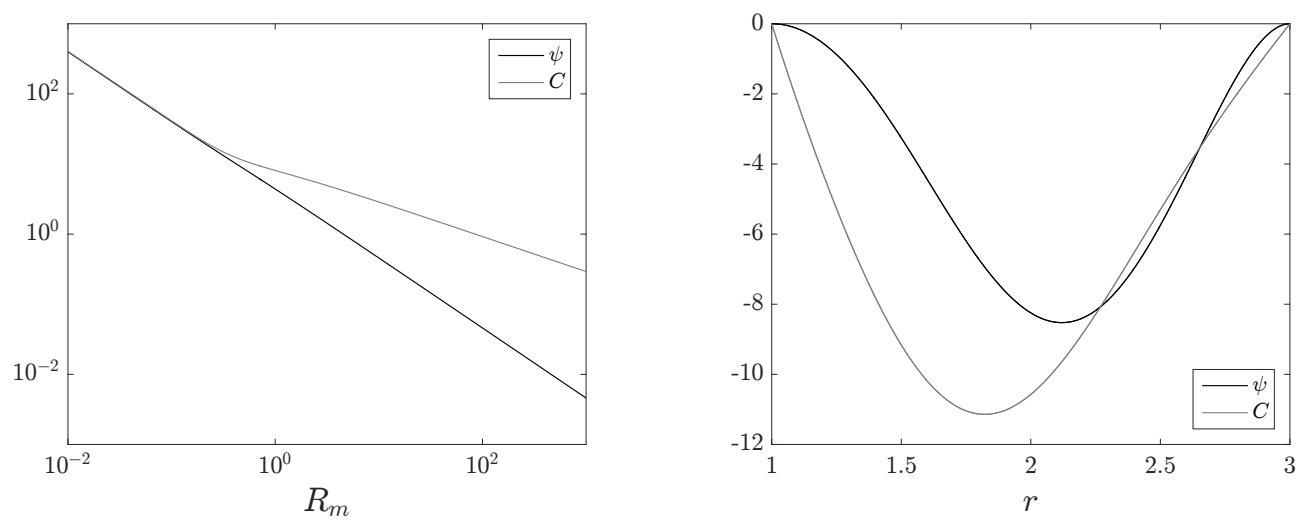

Figure 6. $\quad L^{\infty}$-norm of $\psi$ and $C$ as a function of $R_{m}$. We have that $\psi \sim R_{m}^{-1}$ for all $R_{m}$, whereas $C$ has two distinct regimes, $C \sim R_{m}^{-1}$ as $R_{m} \rightarrow 0$ and $C \sim R_{m}^{-1 / 2}$ as $R_{m} \rightarrow \infty$. On the right are the $\psi$ and $C$ profiles for $R_{m}=0.5$.

point shortly after at $R_{m}=2.27$. Decreasing $R_{m}$ then yields a second solution which merges with the solution branch with $\psi$ and $C$ non-zero and $w=\chi \equiv 0$ at $R_{m}=0.5$. The bifurcation diagram is presented in figure 7 , as well as solution profiles for the lower branch at $R_{m}=2.22$. In addition to this solution branch we find several others with $\chi \equiv 0$ for $R_{m} \sim 1$. This
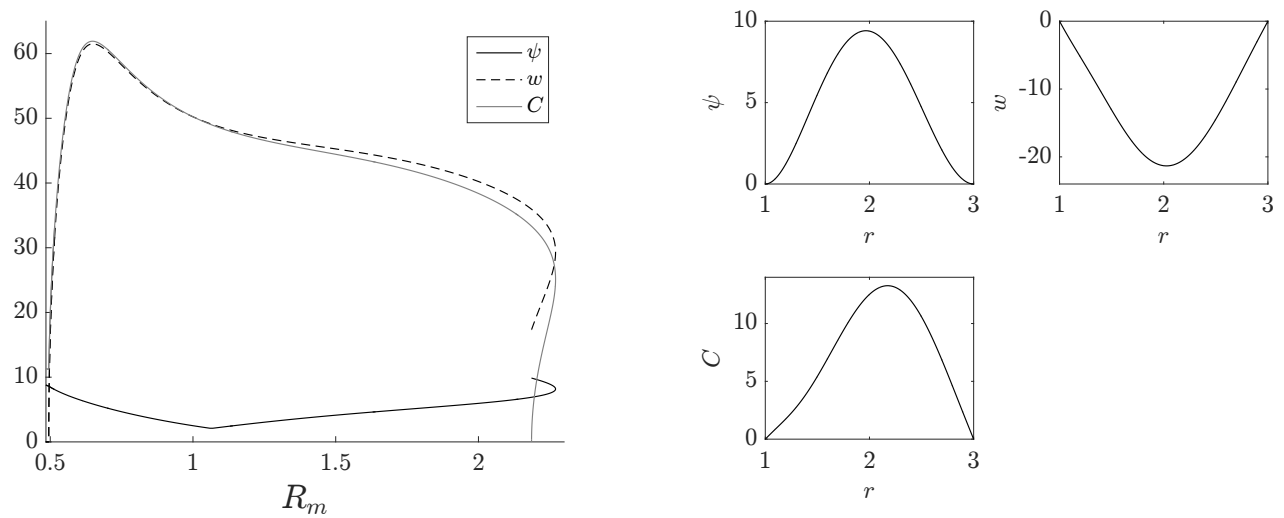

Figure 7. Bifurcation diagram showing $L^{\infty}$-norm of the decoupled solution with $\psi, w$ and $C$ non-zero and $\chi \equiv 0$ as a function of $R_{m}$. This solutions first appears at $R_{m}=2.19$ as predicted by the kinematic equations. It has a turning point at $R_{m}=2.27$ and it merges with another solution branch at $R_{m}=0.5$. On the right we have the solution profiles for $R_{m}=2.22$, for the lower branch (before the turning point). On the upper branch we have $w \simeq C$ over much of the range.

class of solutions is characterised by a dominant balance with $C \simeq w$. A bifurcation diagram for this family of solutions is presented in figure 8, as well as solution profiles for a case with $R_{m} \simeq 1$ marked with a circle. For conciseness we only display the $L^{\infty}$-norm of $C$. This solution family only exists for $R_{m}$ close to 1 , as both viscous and magnetic diffusion play a part in the equilibrium.

There are also other solution branches with $(\psi, C, w)$ non-zero which are omitted from figure 8. These can extend towards $R_{m} \rightarrow 0$ or when $R_{m} \rightarrow \infty$. In the first case a hydrodynamic boundary layer develops, whereas in the latter it is a magnetic boundary layer. Examples of both cases are presented in figure 9 . In this context, we recall that $R_{m}=\nu / \eta$ is a Prandtl number and governs the relative thicknesses of viscous and magnetic layers.

The last type of solution that we find has all 4 fields non-zero. The bifurcation diagram for the $L^{\infty}$-norm of this solution branch is presented in figure 10, as well as solution profiles for $R_{m}=0.3$. The fully coupled solution bifurcates from the zero magnetic field case at 

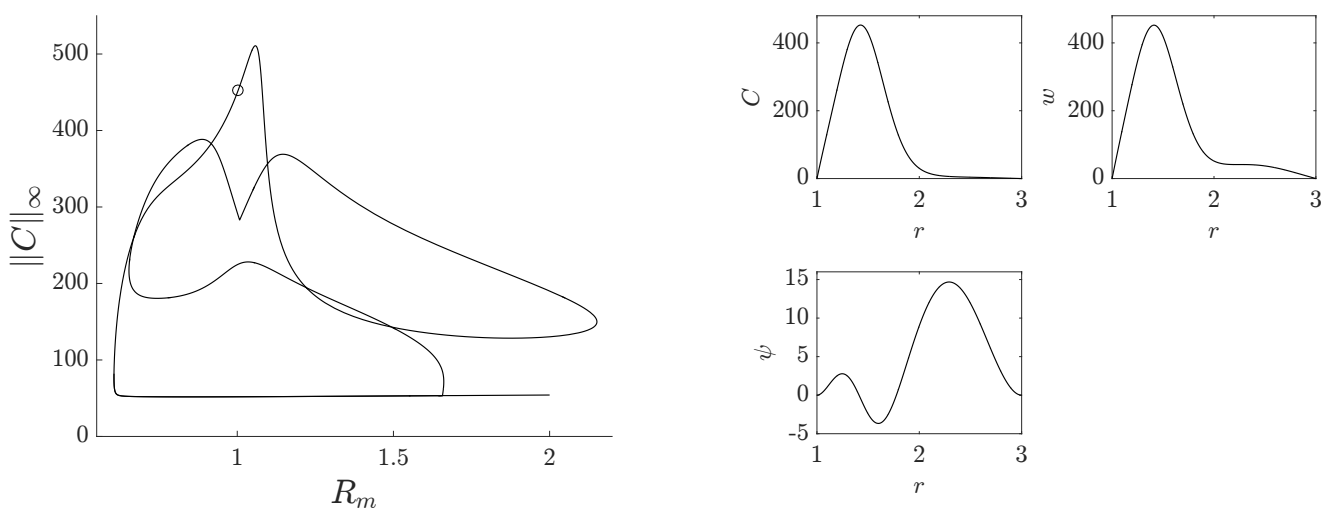

Figure 8. Bifurcation diagram for a class of solutions that only exist for $R_{m} \sim 1$, the selected measure is the $L^{\infty}$-norm of $C$. On the right we have variable profiles for the point marked with a circle. It is clear that $C \simeq w$ through most of the domain.
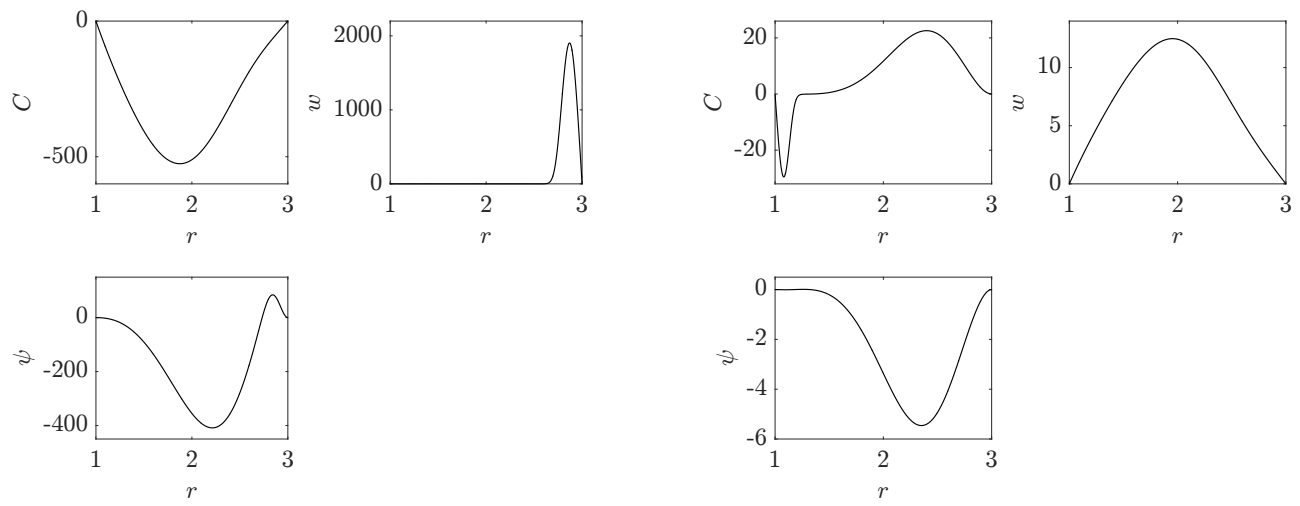

Figure 9. On the left we have a solution with a hydrodynamic boundary layer at the outer wall for $R_{m} \simeq 0.01$. On the right there is a solution with a magnetic boundary layer at the inner wall for $R_{m} \simeq 8000$.

$R_{m}=0.17$, as predicted by the kinematic equations. After a turning point at $R_{m}=0.45$, the upper branch continues back towards $R_{m}=0$. As $R_{m} \rightarrow 0$, we find $\chi \sim w \sim R_{m}^{-3 / 2}$ whereas $\psi \sim C \sim R_{m}^{-1}$. This solution has a viscous boundary layer on the outer wall. Noticeably, we find no equilibria with all variables non-zero for $R_{m}>0.5$ So for large $R_{m}$, the only saturated solutions have $\chi \equiv 0$, which may reflect the difficulty in generating axisymmetric poloidal magnetic field.

\section{Summary and concluding remarks}

The existence of "unforced" hydrodynamic similarity solutions in an annulus is a recently discovered curiosity. Their energy derives from advection from $y= \pm \infty$ and arguably they describe local flow behaviour when shear is imposed at $y= \pm \infty$. We have demonstrated that these unforced flows can give rise also to unforced magnetic fields with a comparable spatial structure. One growing mode is purely azimuthal $(\chi \equiv 0)$, while another has both poloidal and toroidal components, with the growth rate determined by the former. As $R_{m} \rightarrow \infty$ the growth rate $\lambda$ decays as $R_{m}^{-1 / 3}$. In this limit, $\lambda$ is determined by a layer on the outer wall.

The growing magnetic field can equilibrate with the velocity in a number of ways, for all values of $R_{m}$, including subcritical ones. Equilibria exist even as $R_{m} \rightarrow 0$, with all fields 

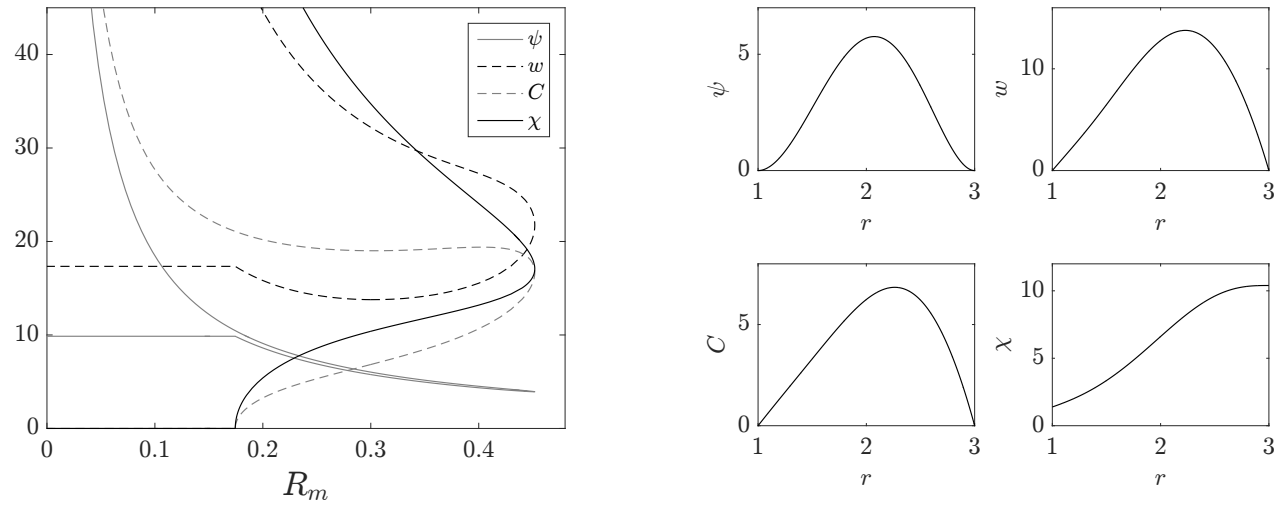

Figure 10. Bifurcation diagram showing $L^{\infty}$-norm of the fully coupled solution as a function of $R_{m}$. The kinematic analysis predicts the starting point $R_{m}=0.17$. On the right we have the solution profiles for $R_{m}=0.3$, on the lower branch (before the turning point).

apparently taking large values, with $|\boldsymbol{u}| \sim R_{m}^{-1}$. However, this is misleading, as it indicates that the appropriate velocity scale is $\eta / L$ rather than $\nu / L$ as $\nu \rightarrow 0$. The solutions in this limit are finite.

A wide variety of solutions exists when $R_{m} \sim 1$, and viscous and magnetic diffusion are comparable. A class of solutions has a dominant balance in the azimuthal vorticity equation with $C \simeq w$ in (18d). This uncommon balance is a result of the similarity scaling and reflects that the $y$-derivative of the magnetic pressure is balancing the $y$-derivative of centripetal acceleration. Comparison of the similar equations (18b) and (18c) indicates that $\psi$ and $R_{m}$ have to deal with the small differences between $C$ and $w$. It is certainly plausible that this should only be possible when $R_{m}$ is close to unity. This behaviour is reminiscent of Cameron and Galloway (2006) where the study of the Archontis dynamo reveals a solution structure with $u \sim B$ when $\eta \sim \nu$.

The unforced hydrodynamic flows are arguably to be expected locally when there is largescale velocity shear at large distances. These we have shown to give rise to dynamo action if the magnetic boundary conditions we have used are applicable. In some circumstances, fields proportional to $y$ would not be permitted and a more involved analysis would be necessary. We believe dynamos would still occur for large $R_{m}$ even were the precise similarity structure lost.

As the steady saturated solutions exhibit a complex structure, we have not investigated the nonlinear time-dependent problem, although this would be a straightforward extension, which might shed light on some of the solutions at high $R_{m}$. In this context, we should not exclude the possibility of solutions exhibiting finite-time blow-up similar to that described in Childress et al. (1989). In our previous work Vaz et al. (2018) we found that for the thermally modified Dean equations in a weakly curved channel there were additional solutions with, for some $t_{0}, \psi, w \propto\left(t_{0}-t\right)^{-1}$ away from the walls, together with shrinking boundary layers. It is possible that similarly divergent hydromagnetic solutions could exist also, but we have not pursued this, as our concern in this paper is on well-behaved flows.

It would also be possible to consider the effect of perfectly conducting rather than insulating exteriors, although we do not believe this would make much qualitative difference. Finally, it should be remembered that we have not examined the stability of our solutions to perturbations of a non-similarity nature. Nevertheless, we believe these local unforced dynamos and hydromagnetic equilibria are of general interest and may be relevant to larger scale problems. 


\section{References}

Allgower, E.L. and Georg, K., Continuation and path following. Acta Numerica, 1993, 2, 164.

Bassom, A.P. and Gilbert, A.D., Nonlinear equilibration of a dynamo in a smooth helical flow. J. Fluid Mech, 1997, 343, 375-406.

Cameron, R. and Galloway, D., Saturation properties of the Archontis dynamo. MNRAS, 2006, 365, 735-746.

Chan, T.F., Newton-Like Pseudo-Arclength Methods for Computing Simple Turning Points. SIAM J. Sci. and Stat. Comput., 1984, 5, 135-148.

Childress, S., Ierley, G.R., Spiegel, E.A. and Young, W.R., Blow-up of unsteady two-dimensional Euler and Navier-Stokes solutions having stagnation-point form. J. Fluid Mech., 1989, 203, 122.

Cowling, T.G., The Magnetic Field of Sunspots. MNRAS, 1933, 94, 39-48.

Gilbert, A.D., Fast dynamo action in the Ponomarenko dynamo. Geophys. Astrophys. Fluid Dyn., 1988, 44, 241-258.

Roberts, P.H. and Soward, A.M., Dynamo theory. Annu. Rev. Fluid Mech., 1992, 24, 459-512.

Ruzmaikin, A., Sokoloff, D. and Shukurov, A., Hydromagnetic screw dynamo. J. Fluid Mech., 1988, $197,3956$.

Vaz, R.H., Boshier, F.A.T. and Mestel, A.J., 'Unforced' Navier-Stokes solutions derived from convection in a curved channel. J. Fluid Mech., 2018 (submitted).

Wynne, J., The smooth Ponomarenko dynamo. Ph.D. Thesis, Imperial College London, 2017. 